\title{
Polarization-correlation optical microscopy of anisotropic biological layers
}

\author{
A. G. Ushenko, A. V. Dubolazov, V. A. Ushenko, Yu. A. \\ Ushenko, M. Yu. Sakhnovskiy, et al.
}

A. G. Ushenko, A. V. Dubolazov, V. A. Ushenko, Yu. A. Ushenko, M. Yu. Sakhnovskiy, V. N. Balazyuk, O. Khukhlina, K. Viligorska, A. Bykov, A. Doronin, I. Meglinski, "Polarization-correlation optical microscopy of anisotropic biological layers," Proc. SPIE 9971, Applications of Digital Image Processing XXXIX, 99712C (28 September 2016); doi: 10.1117/12.2237926

Event: SPIE Optical Engineering + Applications, 2016, San Diego, California, United States 


\title{
Polarization-correlation optical microscopy of anisotropic biological layers
}

\author{
A.G. Ushenko ${ }^{1}$, A.V. Dubolazov ${ }^{1}$, V.A.Ushenko ${ }^{1}$, \\ Yu.A.Ushenko ${ }^{1}$, M.Yu. Sakhnovskiy ${ }^{1}$, V.N. Balazyuk ${ }^{1}$, O. Khukhlina ${ }^{2}$, K. Viligorska ${ }^{2}$, A. Bykov ${ }^{3}$, \\ A. Doronin ${ }^{3}$, I. Meglinski ${ }^{3}$ \\ ${ }^{1}$ Chernivtsi National University, 2 Kotsyubinsky Str., Chernivtsi, 58012, Ukraine \\ ${ }^{2}$ Bukovinian State Medical University, 3 Theatralnaya Sqr., Chernivtsi, 58000, Ukraine \\ ${ }^{3}$ University of Oulu, P.O. Box 4500, Oulu, Finland \\ a.dubolazov@,chnu.edu.ua
}

\begin{abstract}
The theoretical background of azimuthally stable method of Jones-matrix mapping of histological sections of biopsy of myocardium tissue on the basis of spatial frequency selection of the mechanisms of linear and circular birefringence is presented. The diagnostic application of a new correlation parameter - complex degree of mutual anisotropy - is analytically substantiated. The method of measuring coordinate distributions of complex degree of mutual anisotropy with further spatial filtration of their high- and low-frequency components is developed. The interconnections of such distributions with parameters of linear and circular birefringence of myocardium tissue histological sections are found. The comparative results of measuring the coordinate distributions of complex degree of mutual anisotropy formed by fibrillar networks of myosin fibrils of myocardium tissue of different necrotic states - dead due to coronary heart disease and acute coronary insufficiency are shown. The values and ranges of change of the statistical (moments of the 1 st -4 th order) parameters of complex degree of mutual anisotropy coordinate distributions are studied. The objective criteria of differentiation of cause of death are determined.
\end{abstract}

Keywords: polarization, Fourier optics and signal processing, imaging systems, medical and biological imaging.

\section{PART 1. MUELLER MATRIX FORMALISM AS THE BASIS OF CORRELATION POLARIMETRY}

\subsection{Introduction}

Methods of optical diagnostics of biological tissues (BT) include three main directions:

- Spectral methods ${ }^{1-3}$ based on investigation of absorption spectra of biological objects and media.

- Polarization methods ${ }^{4-7}$. The main disadvantage of polarization methods consists in the azimuthal dependence of the obtained data on the rotation angle of the sample in relation to the probing beam ${ }^{8-12}$. Therefore the results of polarization mapping are hard to reproduce.

- The correlation "two-points" methods are devoid of this disadvantage ${ }^{13-15} \cdot \operatorname{In}^{16}$ for characterizing the consistency between the polarization states the object field in the points $\left(d_{1}, d_{2}\right)$ with the intensities $I\left(d_{1}, t\right)$, $I\left(d_{2}, t\right)$ a new azimuthally stable parameter - complex degree of mutual polarization (CDMP) $W\left(d_{1}, d_{2}, t\right)$ is introduced

$$
W\left(d_{1}, d_{2}\right)=\frac{\left(E_{x}\left(d_{1}\right) E^{*}{ }_{x}\left(d_{2}\right)+E_{y}\left(d_{1}\right) E^{*}{ }_{y}\left(d_{2}\right)\right)^{2}}{I\left(d_{1}\right) I\left(d_{2}\right)} .
$$

Applications of Digital Image Processing XXXIX, edited by Andrew G. Tescher, Proc. of SPIE Vol. 9971, 99712C · C 2016 SPIE · CCC code: 0277-786X/16/\$18 · doi: 10.1117/12.2237926 
For practical use in the field of investigation of optically anisotropic tissues one should use following form of equation $(1)^{17-20}$

$$
\operatorname{Re}\{W\} \equiv \widetilde{W}\left(d_{1}, d_{2}\right)=\frac{\left\{E_{x}\left(d_{1}\right) E_{x}\left(d_{2}\right)-E_{y}\left(d_{1}\right) E_{y}\left(d_{2}\right) \cos \left[\theta\left(d_{1}\right)-\theta\left(d_{2}\right)\right]\right\}^{2}}{I\left(d_{1}\right) I\left(d_{2}\right)}
$$

were $\theta\left(d_{1}\right)$ и $\theta\left(d_{2}\right)$ - the phase shifts between the orthogonal components $E_{x}, E_{y}$ of laser beam amplitude.

$\operatorname{In}^{17-20}$ equation (2) takes into consideration linear birefringence of fibrillar networks of biological tissues. Along with this topical task is taking into account of circular birefringence of protein molecules, which form fibrillar networks. The technique proposed $\mathrm{in}^{21}$ is able to separate manifestations of such anisotropy mechanisms by means of usage the technique of direct and inverse Fourier transform of microscopic images of these objects.

This research presents the possibilities of diagnostics of myocardium necrotic changes. At present time postmortem diagnostics of acute coronary syndrome is a leading approach in morphological ${ }^{22,23}$ and forensic ${ }^{24,25}$ investigations. Balanced accuracy ${ }^{26-41}$ of acute coronary insufficiency detection by conventional histochemical methods do not exceed $65 \%-70 \%$ and takes relatively long time. Therefore, topical is the development of new, more accurate and express methods utilizing of novel optical-physical approaches to formation and processing of microscopic images of myocardium histological sections.

This work is aimed to develop and substantiate the method of "two-point" Jones-matrix mapping and spatial-frequency filtering of manifestations of optical anisotropy caused by the necrotic changes of histological sections of myocardium tissue of those who have died of ischemic heart disease (IHD) and acute coronary insufficiency (ACI).

\subsection{Theory}

Biological tissues simultaneously unite two following types of optical anisotropy ${ }^{11}$ :

- phase anisotropy (linear and circular birefringence);

- amplitude anisotropy (linear and circular dichroism).

Optical manifestations of such mechanisms are connected with morphological structure of biological tissues. In this way circular birefringence and dichroism are connected with spiral-like structure of protein molecules. Similarly linear birefringence and dichroism are cause of forming by such molecules of spatially ordered fibrillar networks.

Mechanisms of phase and amplitude anisotropies can be separated by choosing of appropriate spectral region. Protein absorption is minimal in longwave spectral region. Due to this in our spectral region of emission of He-Ne laser ( $\lambda=0.6328 \mu \mathrm{m})$ optical anisotropy of myocardium can be represented by combination of circularly birefringent myosin molecules, which form linearly birefringent fibers and bundles of fibrillar network.

Let consider in details the analytical description of the processes of transformation of laser radiation by myocardium based on the following assumptions ${ }^{6-12}$ :

- myocardium tissue consists of two optically anisotropic components with different scales of spatial organization;

- "small-scale" ( $l \sim 5 \mu m \div 15 \mu m)$ complexes of optically anisotropic molecules of myosin with prevailing circular birefringence;

- "large-scale" fibrillar optically anisotropic network of myosin (range of cross sizes $l \sim 50 \mu m \div 200 \mu m$ ) with prevailing (due to ordering of packing that determines the directions of optical axes of partial biological crystals) linear birefringence;

- polarization properties of the points of such optically anisotropic are characterized by the generalized matrix of optical anisotropy $\{F\}$

$$
\{F\}=\{L\}\{C\}
$$

where $\{L\}$ - Jones matrix of linear birefringence; $\{C\}$ - Jones matrix of circular birefringence or optical activity ${ }^{20}$ 


$$
\left\{\begin{array}{l}
f_{11}=\left[\sin ^{2} \rho+\cos ^{2} \rho \exp (-i \omega)\right] \cos \psi+[\sin \rho \cos \rho(1-\exp (-i \omega))] \sin \psi \\
f_{12}=-\left[\sin ^{2} \rho+\cos ^{2} \rho \exp (-i \omega)\right] \sin \psi+[\sin \rho \cos \rho(1-\exp (-i \omega))] \cos \psi \\
f_{21}=[\sin \rho \cos \rho(1-\exp (-i \omega))] \cos \psi+\left[\cos ^{2} \rho+\sin ^{2} \rho \exp (-i \omega)\right] \sin \psi \\
f_{22}=-[\sin \rho \cos \rho(1-\exp (-i \omega))] \sin \psi+\left[\cos ^{2} \rho+\sin ^{2} \rho \exp (-i \omega)\right] \cos \psi
\end{array}\right.
$$

here $\rho$ - direction of optical axis, $\omega=(2 \pi / \lambda) \Delta n p$ - value of phase shift between the orthogonal components of the amplitude of the laser wave with wavelength $\lambda$, which passed the geometrical path $p$ through the biological crystal with linear birefringence $\Delta n ; \omega$ - rotation angle of the polarization plane of the laser wave caused by circular birefringence.

Taking into account (4) for azimuthally stable situation of biological tissue probing by circularly polarized beam we gain the interrelation between the optical anisotropy of the tissue and the polarization structure of its image.

$$
\left.\left(\begin{array}{c}
E_{x}(d) \\
E_{y}(r)
\end{array}\right)=\frac{1}{\sqrt{2}} \| \begin{array}{ll}
f_{11}(d) & f_{12}(d) \\
f_{21}(d) & f_{22}(d)
\end{array}\right) \|\left(\begin{array}{l}
1 \\
i
\end{array}\right)=\frac{1}{\sqrt{2}}\left(\begin{array}{l}
f_{11}(d)+i f_{12}(d) \\
f_{21}(d)+i f_{22}(d)
\end{array}\right)
$$

Relation (12) can be rewritten as a "single-point" interconnection of the amplitude and optically anisotropic parameters $\left\{E_{x}(d)=g(\rho, \omega, \psi)\right.$; $\left\{U_{y}(d)=j(\rho, \omega, \psi)\right.$.

Within the "two-point" approach ${ }^{16-19}$ similarly to the CDMP parameter $W\left(d_{1}, d_{2}\right)$ (1) the Jones-matrix correlation parameter $H\left(d_{1}, d_{2}\right)$ - the complex degree of mutual anisotropy (CDMA) - can be introduced. It directly characterizes the degree of consistency of linear and circular birefringence of the points of the biological tissue $W\left(\begin{array}{l}E_{x}\left(d_{1}, d_{2}\right) \\ E_{y}\left(d_{1}, d_{2}\right)\end{array}\right) \rightarrow H\left(\begin{array}{c}g\left(f_{i k}\left(d_{1}, d_{2}\right)\right) \\ j\left(f_{i k}\left(d_{1}, d_{2}\right)\right)\end{array}\right)$. Therefore, for determination the analytical expression for CDMA we need use the relation (1) and take into account the equations (3) - (5)

$$
H\left(d_{1}, d_{2}\right)=\frac{\left\{f_{11}\left(d_{1}\right) f_{11}\left(d_{2}\right)+f_{12}\left(d_{1}\right) f_{12}\left(d_{2}\right)+f_{21}\left(d_{1}\right) f_{21}\left(d_{2}\right)+f_{22}\left(d_{1}\right) f_{22}\left(d_{2}\right)\right\}^{2}}{I\left(d_{1}\right) I\left(d_{2}\right)}
$$

Thus we have obtained the relation for a new correlation parameter $H\left(d_{1}, d_{2}\right)$ that describes the generalized anisotropy of different layers of biological tissues. Let us determine the spatial-frequency filtering for separation of coordinate distributions of CDMA formed by various components of the diagnosed layer of myocardium tissue with linear and circular birefringence. From the viewpoint of medicine, the task of "optical selection" of polarization manifestations of the linear $(\rho, \omega)$ and circular $(\psi)$ birefringence of the network of myosin fibrils of myocardium tissue is topical for the differentiation of the cause of death. The matter is that in the case of death due to ACI - there are necrotic changes of myosin structures on small-scale range. Optically such process reveals in degradation of circular birefringence $(\psi \downarrow)$ of the corresponding fibrillar network. In the case of death due to IHD it is accompanied by orientation changes of largescale fibrillar network with the degradation of linear birefringence $(\omega \downarrow)$. Conventional histological diagnostics and differentiation of such necrotic changes is rather labor-intensive, it requires more time and often appears to be ambiguous. For that let us return to the analysis of interconnections ((1)-(6)) between the polarization structure of the image of biological layer and parameters describing its anisotropy. According to the suggested model of such anisotropy the linear birefringence it typical mainly for large-scale fibrillar networks of myosin fibrils. For small-scale structures of optically active molecules the circular birefringence is typical. Thus spatially high-frequency $(E(\rho, \omega))$ and low- 
frequency $(E(\psi)$ ) components are formed in the image of such an object. With the purpose of a selection of such conditions we have applied the method of spatial-frequency filtration of the polarization inhomogeneous laser images in Fourier plane ${ }^{21}$. The main idea of such approach is that spatial-frequency structure of the Fourier form of laser image of the myocardium tissue is different for its large-scale and small-scale protein structures. Therefore, using spatialfrequency filtration it is mainly possible to isolate either low-frequency (with linear birefringence) or high-frequency (with circular birefringence) components, which by means of Fourier transform can be transformed into respectively "separated" laser images. If we locate the vignetting (transparent $L(\Delta \chi, \Delta \gamma)$ opaque $L^{-1}(\Delta \chi, \Delta \gamma)$ ) diaphragm in the central part of Fourier plane, then by means of reverse Fourier transform the low- and high-frequency components can be restored $\left\{\begin{array}{l}\hat{E}(\rho, \omega, \chi, \gamma)=L(\Delta \chi, \Delta \gamma) E(\chi, \gamma) ; \\ \hat{E}(\psi, \chi, \gamma)=L^{-1}(\Delta \chi, \Delta \gamma) E(\chi, \gamma) .\end{array}\right.$ possible to determine the coordinate distributions of the Jones matrix elements of linear $q_{i k}(\rho, \omega)=F(\hat{U})$ and circular $a_{i k}(\psi)=F(\dot{U})$ birefringence according to classical technique ${ }^{20}$. We result in obtaining analytical expressions of the CDMA parameter for various types of optically anisotropic layers of myocardium tissue $H\left(\rho, \omega, d_{1}, d_{2}\right)$ and $H\left(\psi, d_{1}, d_{2}\right)$.

Experimental confirmation of the efficiency of spatial-frequency separation of optical manifestations of mechanisms of phase anisotropy of different scale structures of myocardium is represented by the symmetry of Jones matrix. Thus, for the linear birefringence all matrix elements $f_{i k} \neq 0$ (object field is formed by elliptically polarized parts). For circular birefringence one can see following: $f_{12 ; 21} \rightarrow 0 ; f_{11} \approx f_{22}$ (object field is formed by linearly polarized parts).

\subsection{Optical Realization of Spatial-Frequency Filtering of Coordinate Distributions of CDMA}

Experimental investigations of CDMA coordinate distributions were performed in the classical polarimetry setup ${ }^{5}$. Fig. 1 presents a modified setup of such laser Stokes-polarimeter with spatial-frequency filtration.

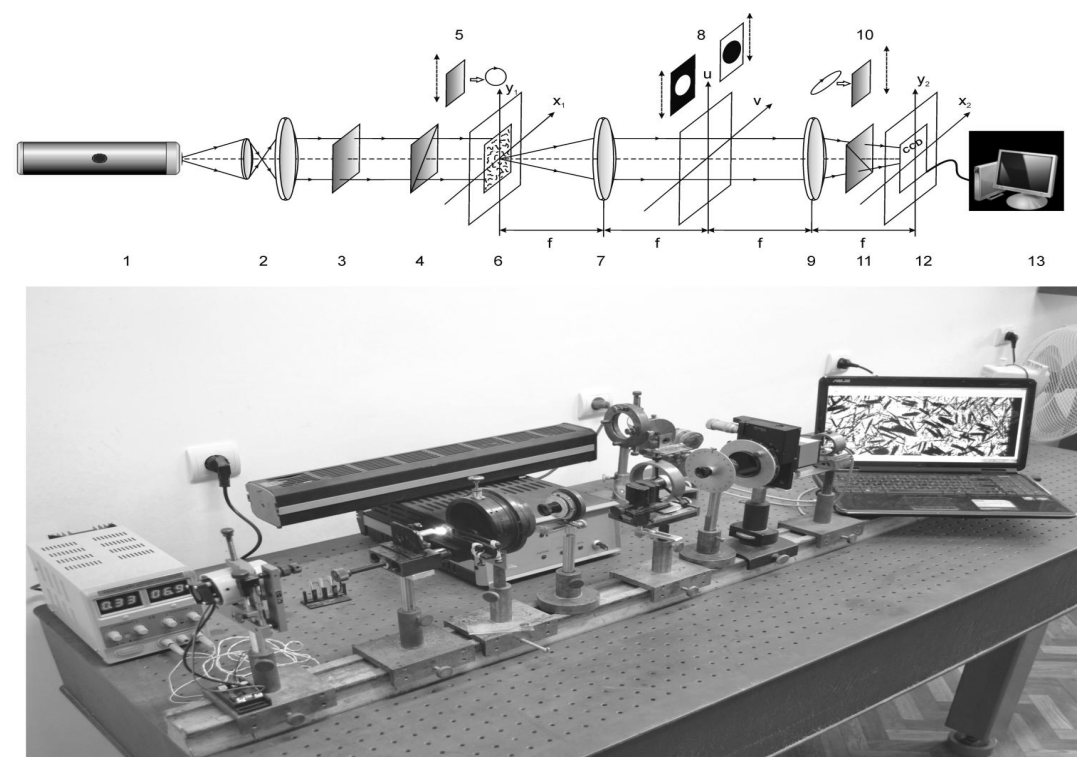

Fig. 1. Optical scheme of Stokes-polarimeter with the use of spatial-frequency filtration where 1 - He-Ne laser; 2 - collimator; 3 stationary quarterwave plate; 5,10 - mechanically movable quarterwave plates; 4,11 - polarizer and analyzer respectively; 6 - object of investigation; 7, 9 - polarization microobjectives; 8 - low-frequency and high-frequency filters, 12 - CCD camera; 13 - personal computer 
Illumination of the investigated sample 6 was performed by parallel $\left(\varnothing=10^{4} \mu \mathrm{m}\right) \mathrm{He}-\mathrm{Ne}(\lambda=0.6328 \mu \mathrm{m}$; power $W=5$ $\mathrm{mW}$ ) laser beam 1, 2. The polarization light source consists of quarter-wave plates 3, 5 (Achromatic True Zero-Order Waveplate) and polarizer 4; this enables to form a laser beam with random azimuth $0^{0} \leq \alpha_{0} \leq 180^{\circ}$ or ellipticity $0^{0} \leq \beta_{0} \leq 90^{0}$ of polarization. Histological sections of miocardium 6 were located in the focal plane of polarization microobjective 7 (Nikon CFI Achromat P, focal distance - 30mm, $N A-0.1$, magnification - 4x). In the back focal plane a spatial-frequency (a low-frequency $L(\Delta \chi, \Delta \gamma)$ or high-frequency $L^{-1}(\Delta \chi, \Delta \gamma)$ one) filter 8 was placed. Polarization microobjective 9 was located at the focal distance from frequency plane of objective 7 and, therefore, performed an inverse Fourier-transform of spatial-temporally filtered field of laser radiation. The coordinate distribution of intensity of such field was registered in the plane of light-sensitive CCD-camera 12 (The Imaging Source DMK 41AU02.AS, monochrome 1/2" CCD, Sony ICX205AL (progressive scan); resolution - 1280x960; size of light-sensitive plate $-7600 \times 6200 \mu \mathrm{m}$; sensitivity $-0.05 \mathrm{~lx}$; dynamic range $-8 \mathrm{bit}$; SNR -9 bit, deviation of photosensitive characteristics from the linear one does not exceed 12\%) that was also located at the focal distance from microobjective 9 and provided the measurement range of the structural elements of the restored image of myocardium tissue histological sections for geometrical sizes $2-2000 \mu \mathrm{m}$.

\section{PART 2. POLARIZATION-CORRELATION MAPS OF BIOLOGICAL LAYERS}

\subsection{Materials and methods}

The experimental measurement of CDMA components - complex elements $d_{i k}$ of Jones matrix - is based on the approach suggested in $^{19}$.

\section{Determination of statistically reliable representative selection of patients with the known (referent) diagnosis}

As objects of investigation we have used optically thin (attenuation coefficient $\tau \approx 0.091 \div 0.097$ ) histological sections of biopsy of myocardium tissue. In this case in the volume of such layers single scattering regime is realized. In other words there is no depolarization (multiple scattering) but transformation of polarization, which is accompanied by formation of polarization-inhomogeneous images of myocardium samples.

From the medical point of view there were formed two groups of patient with the following diagnoses, prepared according to standard technique on the freezing microtome:

- $\quad$ group 1 - dead due to IHD;

- group 2 - dead due to ACI.

In our case group 1 was control and group 2 - under investigation.

The differentiation of IHD (group 1) and ACI (group 2) was determined by the gold standard method - biopsy of surgically removed myocardium. By means of software product Statmate for $95 \%$ confidence interval $(p<0.05)$ a reliable quantity of people was determined $-n=51$. The value of representative sampling was verified by the crosscheck method. It was determined that the value of root-mean-square deviation of the average value of statistical moments $M_{i=1 ; 2 ; 3 ; 4}$ does not exceed 0.025 ; this corresponds to the value of statistically reliable confidence interval $p<0.05$.

\section{Samples production}

Optically thin histological sections of myocardium tissue biopsy were prepared according to standard technique on the freezing microtome.

\section{Algorithms of the analysis of CDMA coordinate distributions}

The measured distributions $q \equiv\left\{H_{\rho, \omega}, H_{\psi}\right\}$ were objectively assessed within the statistical approach - the set of statistical moments of the $1^{\text {st }}-4^{\text {th }}$ order was determined 


$$
M_{1}=\frac{1}{S} \sum_{j=1}^{S} q_{j} ; M_{2}=\sqrt{\frac{1}{S} \sum_{j=1}^{S}\left(q-M_{1}\right)_{j}^{2}} ; M_{3}=\frac{1}{M_{2}{ }^{3}} \frac{1}{S} \sum_{j=1}^{S}\left(q-M_{1}\right)_{j}^{3} ; M_{4}=\frac{1}{M_{2}} \frac{1}{S} \sum_{j=1}^{S}\left(q-M_{1}\right)_{j}{ }^{4}
$$

Here $S$ - number of pixels of CCD-camera. These parameters characterize the mean $\left(M_{1}\right)$, dispersion $\left(M_{2}\right)$, skewness $\left(M_{3}\right)$ and kurtosis or "peak sharpness" $\left(M_{4}\right)$ of histograms $N(q)$.

4.Jones-matrix mapping with spatial-frequency selection of CDMA coordinate distributions of myocardium histological sections.

In the base of experimental measurement of Jones-matrix elements we have used an approach proposed in classical monography of Gerrard and Burch. There Jones matrix consists of two components - real and imaginary. The values of real components of Jones matrix elements can be measured as follows:

- sample was illuminated by linearly polarized beam with azimuth $0^{0}$;

- transmission plane of analyzer was rotated by angles of $0^{0} ; 90^{0}$ and the corresponding intensities of transmitted radiation were measured;

- sample was illuminated by linearly polarized beam with azimuth $90^{\circ}$;

- transmission plane of analyzer was rotated by angles of $0^{0} ; 90^{0}$ and the corresponding intensities of transmitted radiation were measured;

- real components of Jones matrix elements were calculated.

The values of imaginary components of Jones matrix elements can be measured as follows:

- sample was illuminated by right circularly polarized beam (angle between the transmission plane of polarizer and fast axis of quarterwave plate was $45^{0}$ );

- transmission plane of analyzer was rotated by angles of $0^{0} ; 90^{0}$ and the corresponding intensities of transmitted radiation were measured;

- sample was illuminated by linearly polarized beam with azimuth $45^{\circ}$;

- transmission plane of analyzer was rotated by angles of $0^{0} ; 90^{0}$ and the corresponding intensities of transmitted radiation were measured;

- imaginary components of Jones matrix elements were calculated.

For the purpose of choosing of diaphragm size the dependencies of values of all statistical moments from the size of spatial-frequency diaphragm were investigated. It was determined that beginning from the specific size of spatialfrequency filter ( $\Delta R=125$ pixels) the change of values of the set of statistical moments reached its saturation. As the criterion of optimal size we chose the condition of simultaneous extremal values of all statistical moments. For lowfrequency filter (transparent) the diaphragm size was $\Delta \mathrm{R}=30$ pixels - transmission of spatial frequencies up to $v=10$ $\mathrm{mm}^{-1}$. Optimal size of high-frequency filter (opaque) was $\Delta R^{-1}=60$ pixels - transmission of spatial frequencies from $v$ $=20 \mathrm{~mm}^{-1}$.

Efficiency of spatial-frequency filtering of manifestations of linear and circular birefringence was estimated in accordance with the symmetry of Jones matrixes and prevailing polarization type high- and low-frequency components of images of myocardium samples.

It was determined that in the case of low-frequency filtering all elements of Jones matrix $f_{i k} \neq 0$. In addition, $94 \%$ of the pixels of digital camera recorded elliptically polarized areas of the myocardium image. In the case of high-frequency filtering the following results were obtained - $f_{12 ; 21} \rightarrow 0 ; f_{11} \approx f_{22}$ ( $91 \%$ of linearly polarized areas).

The values of CDMA were calculated as follows:

- under different diaphragming conditions the two-dimensional arrays of values of Jones matrix elements were measured within each pixel;

- for each line in two-dimensional array of Jones matrix elements a pairs of neighbor pixels were chosen;

- for each pair of such pixels one value of CDMA was calculated;

- at the next stage by means of scanning with the step of one pixel a set of CDMA values was determined within the chosen line of CCD camera; 
- above mentioned scanning was performed within all lines of light sensitive area of CCD camera and the twodimensional array of CDMA values was calculated.

\subsection{Polarization-correlation maps}

The figures 2 and 3 present the results of Jones-matrix mapping of low-frequency (linear birefringence of fibrillar networks - Fig. 2) and high-frequency (circular birefringence of myosin molecules - Fig. 3) CDMA distributions of myocardium tissue histological sections. Figures consists of the coordinate distributions (fragments (1),(3)) and histograms $N(q)$ (fragments (2),(4)) of sample randomly taken from group 1 (fragments (1),(2)) and from group 2 (fragments (3),(4)).

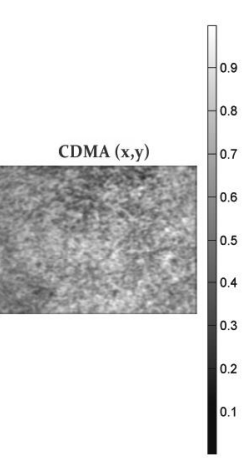

1)

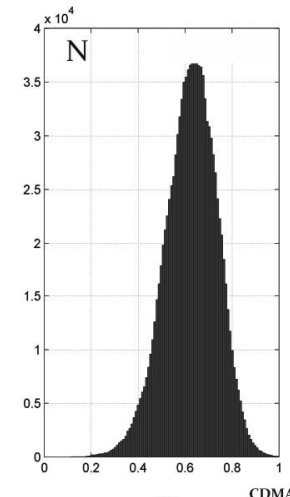

2)

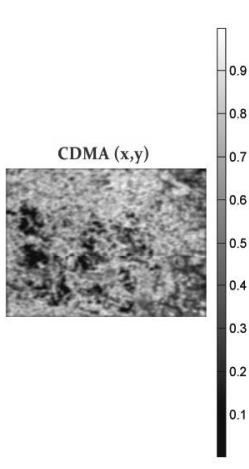

3)

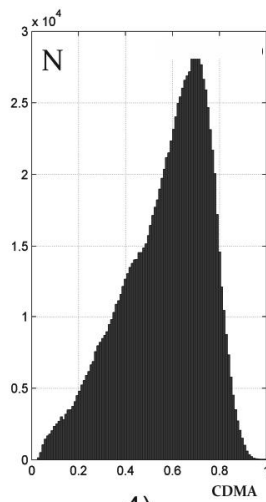

4)

Fig. 2. Coordinate structure ((1),(3)) and histograms ((2),(4)) of CDMA distributions of linear birefringence of histological sections of biopsy of myocardium tissue of group $1((1),(2))$ and group $2((3),(4))$.

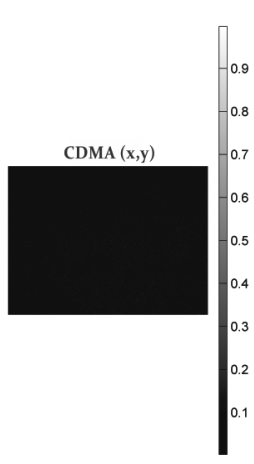

1)

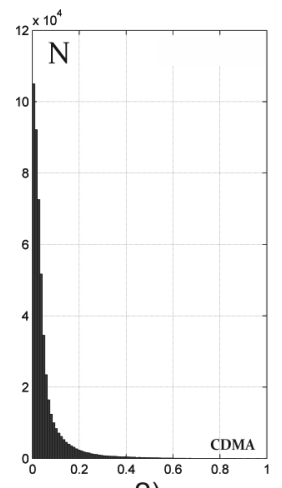

2)

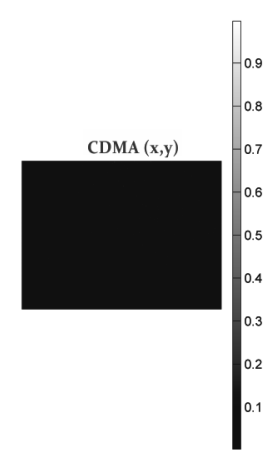

3)

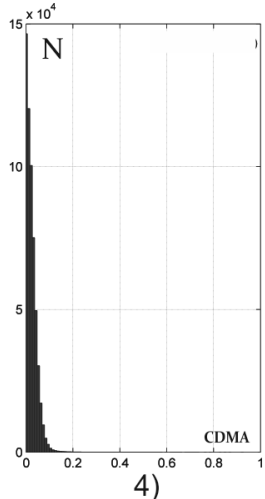

4)

Fig. 3. Coordinate structure ((1),(3)) and histograms ((2),(4)) of CDMA distributions of circular birefringence of histological sections of biopsy of myocardium tissue of group $1((1),(2))$ and group $2((3),(4))$.

$\boldsymbol{C D M A} H_{\rho, \omega}$. The comparative analysis of the spatial-frequency filtered coordinate distributions $H_{\rho, \omega}(m \times n)$ of large-scale network of myocardium layer revealed certain difference between them. The histograms of the CDMA distribution for a histological section of myocardium from group 2 (ACI) are characterized by asymmetric structure (Fig. 2, fragment (4)). The similar distribution found for the tissue sample from group 1 (IHD) (Fig. 2, fragment (2)) is more symmetrical. The revealed peculiarity is related to the structure of myosin fibrils. For histological sections of group 2 the directions of the myosin fibrils are more ordered in the direction of optical axes. For the samples from group 1 the similar network of myocardium layer is more disordered in directions. Optically this geometrical structure $\left(\rho^{*} \leftrightarrow N_{\max }\right)$ reveals in the formation of the priority, most probable values of CDMA $H_{\rho, \omega}(m \times n)$ (relations (1), (3)-(6)), the set of which forms the main extreme. Statistically such a process is detected by the following changes of 
$M_{i=1 ; 2 ; 3 ; 4}\left(H_{\rho, \omega}\right)$ (relations (7)). For the IHD - the decrease of the skewness $M_{3} \downarrow$ and the kurtosis $M_{4} \downarrow$ of histograms $N\left(H_{\rho, \omega}\right)$. Other statistical moments (mean $M_{1} \uparrow$, dispersion $M_{2} \uparrow$ ) increase. Thus "CDMA $H_{\rho, \omega}$ biopsy" appeared to be sensitive to the changes of linear birefringence caused by necrotic transformation of fibrillar networks of myocardium. That is why this information is important for differentiation of causes of death due to IHD and ACI.

CDMA $H_{\psi}$. The physical mechanism of circular birefringence formation is first of all related to concentration of optically active protein molecules of myosin, which form the myocardium fibrillar networks. Necrotic changes caused by ACI lead to decrease of concentration of protein molecules. Thus the correlation parameter $W_{\theta}$ of such layers is characterized by the less magnitude of values in comparison with the CDMA distribution obtained for myocardium from group 1. Quantitatively such difference characterizes histograms $N\left(H_{\psi}\right)$ (Fig. 3, fragment (2)). It is obvious from the obtained data (Fig. 3, fragment (4)) that for necrotic changes caused by ACI less in magnitude values of the average $M_{1}\left(H_{\psi}\right) \downarrow$, dispersion $M_{2}\left(H_{\psi}\right) \downarrow$ are typical. The skewness $M_{3}\left(H_{\psi}\right) \uparrow$ and the kurtosis $M_{4}\left(H_{\psi}\right) \uparrow$ of distributions $N\left(H_{\psi}\right)$ increases. This fact indicates to sensitivity "CDMA $H_{\psi}$ - biopsy" to "concentration" changes of circular birefringence of myocardium. That is why such information is the most important in the ACI diagnostics of myocardium.

\subsection{Statistical intergroup analysis}

The differentiation between the group 1 and group 2 was determined by using of the following methodology ${ }^{26-28}$ :

- within each set of values of statistical moments $M_{i=1 ; 2 ; 3 ; 4}$ we determined the average value $\bar{M}_{i=1 ; 2 ; 3 ; 4}$ and standard deviation $\sigma_{i=1 ; 2 ; 3 ; 4}$;

- differences between the statistical sets $M_{i=1 ; 2 ; 3 ; 4}$ were significant in the case when the average value $\bar{M}_{i=1 ; 2 ; 3 ; 4}$ within the group 1 didn't "overlap" with the standard deviation $\sigma_{i=1 ; 2 ; 3 ; 4}$ within group 2 and vice versa.

- within both groups of myocardium samples for the distributions of values of each statistical moments $M_{i=1 ; 2 ; 3 ; 4}$ we chose cutoff of $3 \sigma\left(99.72 \%\right.$ of all possible values of changes of $\left.M_{i}\right)$. Sequentially, we determined the number of "false negative" ( $b$ ) and "false positive" ( $d$ ) conclusions;

- For every statistical moments traditional for probative medicine operational characteristics: sensitivity $\left(S e=\frac{a}{a+b} 100 \%\right)$, specificity $\left(S p=\frac{c}{c+d} 100 \%\right)$ and balanced accuracy $\left(A c=\frac{S e+S p}{2}\right)$, where $a$ and $b$ are the number of correct and wrong diagnoses within group 2; $c$ and $d$ - the same within group 1 were determined.

Table 1. Parameters of statistical structure of CDMA coordinate distributions

\begin{tabular}{|c|c|c|c|c|}
\hline \multirow{2}{*}{ Parameters } & \multicolumn{2}{|c|}{$H_{\rho, \omega}$} & \multicolumn{2}{c|}{$H_{\psi}$} \\
\cline { 2 - 5 } & IHD & ACI & IHD & ACI \\
\hline$M_{1}$ & $0.78 \pm 0.054$ & $0.71 \pm 0.051$ & $0.07 \pm 0.005$ & $0.05 \pm 0.004$ \\
\hline$M_{2}$ & $0.22 \pm 0.014$ & $0.25 \pm 0.015$ & $0.13 \pm 0.007$ & $0.07 \pm 0.006$ \\
\hline$M_{3}$ & $0.37 \pm 0.023$ & $0.24 \pm 0.016$ & $0.71 \pm 0.057$ & $1.21 \pm 0.084$ \\
\hline$M_{4}$ & $0.63 \pm 0.035$ & $0.76 \pm 0.15$ & $1.28 \pm 0.091$ & $1.98 \pm 0.14$ \\
\hline
\end{tabular}


The comparative analysis of the data obtained (Table 1) showed that the differences between the values of average $\bar{M}_{i=1 ; 2 ; 3 ; 4}$ moments of all orders are statistically reliable. However, there is an intergroup overlap for all histograms $N\left(M_{i}\right)$. Moreover, the range of such an overlap is inversely proportional to the value of the difference between the averages $\bar{M}_{i=1 ; 2 ; 3 ; 4}$. The moments $M_{i=3 ; 4}\left(H_{\rho, \omega}\right)$ appeared to be sensitive in differentiation of linear birefringence maps $H_{\rho, \omega}(m \times n)$ of myocardium histological sections (highlighted in grey in Table 1). For circular birefringence distributions $H_{\psi}(m \times n)$ of myocardium layers the most preferable appear to be statistical moments of higher orders $M_{i=3 ; 4}\left(H_{\psi}\right)$.

Table 2 presents the parameters of information value of azimuthally stable method of Jones-matrix mapping of optical anisotropy of histological sections of biopsy of myocardium with different necrotic changes.

Table 2. Operational characteristics of the method of Jones-matrix mapping of optical anisotropy of histological sections of biopsy of myocardium

\begin{tabular}{|c|c|c|c|}
\hline Parameters & $M_{i}$ & $H_{\rho, \omega}$ & $H_{\psi}$ \\
\hline \multirow{3}{*}{$A c\left(M_{i}\right)$} & $M_{1}$ & $60 \%$ & $71 \%$ \\
\cline { 2 - 4 } & $M_{2}$ & $55 \%$ & $67 \%$ \\
\cline { 2 - 4 } & $M_{3}$ & $95 \%$ & $87 \%$ \\
\cline { 2 - 4 } & $M_{4}$ & $90 \%$ & $93 \%$ \\
\hline
\end{tabular}

The obtained results enable to state a rather high level of accuracy of azimuthally stable Jones-matrix mapping. According to the criteria of probative medicine ${ }^{26}$ the parameters $A c(\psi) \sim 90 \%$ correspond to good quality, while $A c(\rho, \omega)>90 \%$ - to high quality.

\section{CONCLUSION}

The comparative investigations of the effectiveness of the developed technique of spatial-frequency Fourier polarimetry of CDMA in the diagnostics of the necrotic changes of myocardium tissue are carried out.

The criteria of differentiation between the causes of death due to IHD and ACI on the basis of the statistical (statistical moments of the $1^{\text {st }}-4^{\text {th }}$ order) analyses of the spatial-frequency filtered distributions of CDMA of protein networks with linear and circular birefringence are determined.

\section{Acknowledgement}

This work was supported by the grant №0116U001446; №0116U001449; №0115U003241; №0115U003227; №0115U003235 from the Ukraine Foundation for Basic Research.

\section{REFERENCES}

[1]. Tuchin, V.V., [Tissue Optics: Light Scattering Methods and Instruments for Medical Diagnosis], second edition, PM 166, SPIE Press, Bellingham, WA (2007).

[2]. X. Wang, G. Yao, L. - H. Wang, "Monte Carlo model and single-scattering approximation of polarized light propagation in turbid media containing glucose," Appl. Opt. 41, 792-801 (2002).

[3]. X. Wang, L. - H. Wang, "Propagation of polarized light in birefringent turbid media: a Monte Carlo study," J. Biomed. Opt. 7, 279-290 (2002).

[4]. Angelsky, O.V., Bekshaev, A.Ya., Maksimyak, P.P., Maksimyak, A.P., Hanson, S.G., Zenkova, C.Yu. "Selfaction of continuous laser radiation and Pearcey diffraction in a water suspension with light-absorbing particles," Optics Express 22 (3), 2267-2277 (2014).

[5]. W.-F. Cheong, S. A. Prahl, A. J. Welch, "A Review of the Optical Properties of Biological Tissues," IEEE J. of Quantum Electron. 26, 2166-2185 (1990). 
[6]. Angelsky, O.V., Bekshaev, A.Ya., Maksimyak, P.P., Maksimyak, A.P., Hanson, S.G., Zenkova, C.Yu. "Selfdiffraction of continuous laser radiation in a disperse medium with absorbing particles," Optics Express 21 (7), 8922-8938 (2013).

[7]. O. V. Angel'skiı̌, A. G. Ushenko, A. D. Arkhelyuk, S. B. Ermolenko, D. N. Burkovets, "Scattering of laser radiation by multifractal biological structures," Optics and Spectroscopy, 88 (3), 444-447 (2000).

[8]. Angelsky, O.V., Bekshaev, A.Ya., Maksimyak, P.P., Maksimyak, A.P., Hanson, S.G., Zenkova, C.Yu. "Selfaction of continuous laser radiation and Pearcey diffraction in a water suspension with light-absorbing particles," Optics Express 22 (3), 2267-2277 (2014).

[9]. Angelsky, O.V., Bekshaev, A.Ya., Maksimyak, P.P., Maksimyak, A.P., Hanson, S.G. "Measurement of small light absorption in microparticles by means of optically induced rotation," Optics Express 23 (6), 7152 7163 (2015).

[10]. Yu. A. Ushenko, T. M. Boychuk, V. T. Bachynsky, O. P. Mincer, "Diagnostics of Structure and Physiological State of Birefringent Biological Tissues: Statistical, Correlation and Topological Approaches" in Handbook of Coherent-Domain Optical Methods, pp. 107-148, Springer Science+Business Media New York (2013).

[11]. Yu. A. Ushenko, "Investigation of formation and interrelations of polarization singular structure and Muellermatrix images of biological tissues and diagnostics of their cancer changes," J. Biomed. Opt. 16, 066006 (2011).

[12]. Angelsky, O.V., Besaha, R.N., Mokhun, A.I., Mokhun, I.I., Sopin, M.O., Soskin, M.S. "Singularities in vectoral fields," Proc. SPIE, 40-54 (1999).

[13]. E. Wolf, "Unified theory of coherence and polarization of random electromagnetic beams," Phys. Lett. A. 312, 263-267 (2003).

[14]. J. Tervo, T. Setala, A. Friberg, "Degree of coherence for electromagnetic fields," Opt. Express 11, 1137-1143 (2003).

[15]. Angelsky, O.V., Tomka, Y.Y., Ushenko, A.G., Ushenko, Y.G., Yermolenko S.B. "2-D tomography of biotissue images in pre-clinic diagnostics of their pre-cancer states," Proc. SPIE., 5972, 158-162 (2005).

[16]. A. G. Ushenko, P. O. Angelsky, M. Sidor, Yu. F. Marchuk, D. R. Andreychuk, and N. V. Pashkovskaya, "Spatialfrequency selection of complex degree of coherence of laser images of blood plasma in diagnostics and differentiation of pathological states of human organism of various nosology," Appl. Opt. 53, B172-B180 (2014).

[17]. Ushenko, A.G., Burkovets, D.N., Ushenko, Yu.A.” Polarization Phase Mapping and Reconstruction of Biological Tissue Architectonics during Diagnosis of Pathological Lesions," Optics and Spectroscopy (English translation of Optika i Spektroskopiya) 93 (3), 449-456 (2002).

[18]. Yu. A. Ushenko, Yu. Ya. Tomka and A. V. Dubolazov, "Complex degree of mutual anisotropy of extracellular matrix of biological tissues," Optics and Spectroscopy 110, 814-819 (2011).

[19]. A. Gerrard, J. M. Burch, [Introduction to matrix methods in optics], New York.: A Wiley-Interscience Publication (1975).

[20]. Angel'skiǐ, O.V., Ushenko, A.G., Ermolenko, S.B.,Ushenko, Yu.A., Pishak, O.V. "Polarization Based Visualization of Multifractal Structures for the Diagnostics of Pathological Changes in Biological Tissues," Optics and Spectroscopy (English translation of Optika i Spektroskopiya) 89 (5), 799-804 (2000).

[21]. J. W. Goodman, [Statistical properties of laser speckle patters. In: Laser Speckle and Related Phenomena], Ed. J.C. Dainty, pp. 9-75, Berlin: Springer-Verlag (1975).

[22]. L. V. Kakturski1̄, "Clinical morphology of acute coronary syndrome," Arkh Patol. 69, 16-19 (2007).

[23]. Ushenko, V. A., Sidor, M. I., Marchuk, Y. F., Pashkovskaya, N. V., Andreichuk, D. R. "Azimuth-invariant mueller-matrix differentiation of the optical anisotropy of biological tissues," Optics and Spectroscopy, 117(1), 152-157 (2014).

[24]. V.A. Ushenko, N.I. Zabolotna, S.V. Pavlov, D.M. Burcovets, O.Yu. Novakovska, "Mueller-matrices polarization selection of two-dimensional linear and circular birefringence images," Proc. SPIE 9066, Eleventh International Conference on Correlation Optics, 90661X (2013).

[25]. Ushenko, V.A., Gorsky, M.P., "Complex degree of mutual anisotropy of linear birefringence and optical activity of biological tissues in diagnostics of prostate cancer," Optics and Spectroscopy 115(2), 290-297 (2013).

[26]. Cristina Basso, Fiorella Calabrese, Domenico Corrado, Gaetano Thiene, "Postmortem diagnosis in sudden cardiac death victims: macroscopic, microscopic and molecular findings," Cardiovascular Research 50, 290-300 (2001).

[27]. M.D. Pérez-Cárceles, J. Noguera, J.L. Jiménez, P. Martínez, A. Luna, E. Osuna, "Diagnostic efficacy of biochemical markers in diagnosis post-mortem of ischaemic heart disease," Forensic Science International 142, 17 (2004). 
[28]. Ushenko, Y. A., Gorskii, M. P., Dubolazov, A. V., Motrich, A. V., Ushenko, V. A., Sidor, M. I., "Spatialfrequency Fourier polarimetry of the complex degree of mutual anisotropy of linear and circular birefringence in the diagnostics of oncological changes in morphological structure of biological tissues," Quantum Electronics, 42(8), 727, (2012).

[29]. Ushenko, V. A., "Complex degree of mutual coherence of biological liquids," In ROMOPTO International Conference on Micro-to Nano-Photonics III (pp. 88820V-88820V). International Society for Optics and Photonics. (2013).

[30]. F. Martínez Díaz, M. Rodríguez-Morlensín, M.D. Pérez-Cárceles, J. Noguera, A. Luna and E. Osuna, "Biochemical analysis and immunohistochemical determination of cardiac troponin for the postmortem diagnosis of myocardial damage," Histol. Histopathol. 20, 475-481 (2005).

[31]. Angelsky, O.V., Polyanskii, P.V., Hanson, S.G. "Singular optical coloring of regularly scattered white light," Optics Express 14 (17), 75797586 (2006).

[32]. Ushenko, Y. O., Dubolazov, O. V., Karachevtsev, A. O., Gorsky, M. P., Marchuk, Y. F., "Wavelet analysis of Fourier polarized images of the human bile," Applied optics, 51(10), C133-C139, (2012).

[33]. Ushenko, Y. A., Ushenko, V. A., Dubolazov, A. V., Balanetskaya, V. O., Zabolotna, N.I., "Mueller-matrix diagnostics of optical properties of polycrystalline networks of human blood plasma," Optics and Spectroscopy, 112(6), 884-892, (2012).

[34]. Yu A Ushenko, Yu Ya Tomka, A V Dubolazov, "Laser diagnostics of anisotropy in birefringent networks of biological tissues in different physiological conditions," QUANTUM ELECTRON, 41 (2), 170-175, (2011).

[35]. V. A. Ushenko ; G. D. Koval ; M. S. Gavrylyak; "Mueller-matrices polarization selection of two-dimensional linear and circular birefringence images," Proc. SPIE 8856, Applications of Digital Image Processing XXXVI, 88562E (September 26, 2013);

[36]. M. S. Gavrylyak ; P. M. Grygoryshyn; "The correlation-optical method measuring time of thrombus formation," Proc. SPIE 9066, Eleventh International Conference on Correlation Optics, 90661U (December 17, 2013);

[37]. Gavrylyak, M.S., "Correlation method for measuring the largest Lyapunov exponent in optical fields," Ukr. J. Phys. Opt. ,9(2), 119-127 (2008).

[38]. Angelsky, O.V., Ushenko, A.G., Ushenko, Y.G. "Complex degree of mutual polarization of biological tissue coherent images for the diagnostics of their physiological state," Journal of Biomedical Optics 10 (6), 060502 060502-3 (2005).

[39]. C. S. Davis, [Statistical methods of the analysis of repeated measurements], New York: Springer-Verlag, (2002).

[40]. A. Petrie, B. Sabin, [Medical Statistics at a Glance], Blackwell Publishing (2005). 